Preprint typeset in JHEP style - HYPER VERSION

hep-th/0307180

HU-EP-03/40

\title{
Towards the deconstruction of M-theory
}

\author{
Ingo Kirsch and Dan Oprisa \\ Institut für Physik, \\ Humboldt-Universität zu Berlin, \\ Newtonstraße 15, \\ D-12489 Berlin, \\ Germany \\ E-mail: ik@physik.hu-berlin.de, eprisa@physik.hu-berlin.de
}

\begin{abstract}
We argue that there is an equivalence of M-theory on $T^{3} \times A_{N-1}$ with a fourdimensional non-supersymmetric quiver gauge theory on the Higgs branch. The quiver theory in question has gauge group $S U(N)^{N_{4} N_{6} N_{8}}$ and is considered in a strong coupling and large $N_{4,6,8}$ limit. We provide field- and string-theoretical evidence for the equivalence making use of the deconstruction technique. In particular, we find wrapped M2-branes in the mass spectrum of the quiver theory at low energies.
\end{abstract}

KEYWORDS: Deconstruction of extra dimensions, M-theory. 


\section{Contents}

1. Introduction 1

2. Deconstruction of M-theory on $T^{3} \times A_{N-1}$

2.1 Non-supersymmetric orbifolds 3

2.2 D3-branes at $\mathbb{C}^{3} / \mathbb{Z}_{N_{4}} \times \mathbb{Z}_{N_{6}} \times \mathbb{Z}_{N_{8}}$ : orbifold realization of a non-supersymmetric quiver theory

2.3 Generation of three compact extra dimensions in the low-energy effective field theory 9

2.4 M2-branes on the Higgs branch 10

2.5 Summary of the field theory results 10

3. String-theoretical motivation for the equivalence 11

4. Conclusions 13

\section{Introduction}

Deconstruction has found many applications in both phenomenology and string theory since its first use to generate extra dimensions in theories with internal gauge symmetries 11, 2] (for early work on this subject, see [3, 4]). Non-renormalizable theories in $D>4$ are ill-defined above a certain cut-off at which they become strongly coupled due to a coupling constant with negative dimension. At high energies these theories require an ultraviolet completion which can frequently be provided by the deconstruction technique. This UV completion is generically a quiver theory [5] characterized by a discrete theory space, the moose or quiver diagram, which represents the field content of the theory by a lattice of sites and links. In a certain low-energy limit the quiver theory develops one or more extra dimensions and reproduces the higher-dimensional non-renormalizable theory. A peculiarity of the deconstruction approach is that the ultraviolet theory has less dimensions than the infrared theory. This is different from compactified theories which reveal their higher-dimensional behaviour at energies above the inverse radius of the compactified dimension.

In some instances a continuum limit of the quiver theory exists such that the theory is higher-dimensional to arbitrary high energies. In the known examples the continuum theory is a theory which has been predicted to exist by string theory arguments, but which lacks a conventional local Lagrangian description. This has led to the deconstruction of the six-dimensional $(2,0)$ theory and $(1,1)$ little string theory [6] (see also [7]) which describe the decoupling limit of multiple parallel M5- and NS5-branes. Obstructions to finding 
a conventional local Lagrangian description of these theories arise due to difficulties in constructing a non-abelian chiral two-form and/or non-locality. In [6] both theories were deconstructed from four-dimensional quiver theories describing D3-branes at orbifolds of the type $\mathbb{C}^{3} / \mathbb{Z}_{N}$ and $\mathbb{C}^{3} / \mathbb{Z}_{N_{5}} \times \mathbb{Z}_{N_{6}}$. Another interesting theory without a Lagrangian description is that of intersecting M5-branes. The deconstruction of this theory [8, 9] was accomplished by taking a large $N$ limit of the theory describing intersecting D3-branes 10] at an orbifold $\mathbb{C}^{2} / \mathbb{Z}_{N}$. Tensionless strings localized at the intersection were identified in the spectrum of the corresponding $(4,0)$ defect conformal field theory. For further applications of deconstruction in string theory see Refs. [11, 12, 13, 14, 15, 16, 17, 18].

Recently, the deconstruction of extra dimensions in theories with gravity was studied in [19, 20, 21, 22, 23]. The basic idea is to consider $d+1$-dimensional Einstein gravity as the low-energy effective theory of a $d$-dimensional gravitational theory with a discrete theory space. The continuum physics of the $d+1$-dimensional gravitational theory can be reproduced correctly at energies parametrically higher than the compactification scale. However, a peculiar UV/IR connection was found forbidding the deconstruction all the way up to the $d+1$-dimensional Planck scale.

An open problem along these lines is the deconstruction of M-theory itself. In [6] it was proposed to deconstruct M-theory on an $A_{N-1}$ singularity from a particular $(1,0)$ little string theory (LST). This LST is defined as the decoupling limit of $N$ NS5-branes at an orbifold singularity of the type $\mathbb{C}^{2} / \mathbb{Z}_{k}$. A seventh dimension arises on the Higgs branch of this theory. In a continuum $(k \rightarrow \infty)$ limit one expects to obtain a seven-dimensional gauge theory together with its UV completion. Exploiting string dualities for $k \rightarrow \infty$, it was shown [6] that the stack of NS5-branes maps to M-theory on $A_{N-1}$, which is a UV completion of the seven-dimensional gauge theory.

A direct deconstruction seems to be impossible due to the obstructions to finding a (conventional) Lagrangian description for LST. Alternatively, one could first deconstruct the NS5-brane theory out of the D3-brane theory at $\mathbb{C}^{3} / \mathbb{Z}_{N_{5}} \times \mathbb{Z}_{N_{6}}[6]$. One obtains a lattice action for LST which could in principle be orbifolded again by projecting out degrees of freedom which are not invariant under the (second) orbifold $\mathbb{C}^{2} / \mathbb{Z}_{k}$. Here one faces the problem that it is difficult, if not impossible, to find an $S U(2)$ R-symmetry inside the lattice action into which the $\mathbb{Z}_{k}$ orbifold action can be embedded. Note that the $S O$ (4) R-symmetry of the NS5-brane theory is only recovered in the continuum limit. It is therefore not obvious how to further discretize the latticized LST action.

In this paper we apply the deconstruction method to M-theory following a slightly different approach. We deconstruct M-theory directly from a four-dimensional non-supersymmetric quiver gauge theory with gauge group $S U(N)^{N_{4} N_{6} N_{8}}$ and $N_{4,6,8}$ three positive integers. The corresponding orbifold realization is given by a stack of D3-branes in type IIB string theory placed at the origin of $\mathbb{C}^{3} / \Gamma$, where the orbifold group $\Gamma$ is the product of three cyclic groups $\mathbb{Z}_{N_{4}} \times \mathbb{Z}_{N_{6}} \times \mathbb{Z}_{N_{8}}$. These groups generate three circular orbits in the directions 468. The quiver diagram is a three-dimensional body-centred cubic lattice. At a certain point in the moduli space, each of the $\mathbb{Z}_{N}$ factors generates a circular discretized extra dimension. In an appropriate $N_{4,6,8} \rightarrow \infty$ limit, the extra dimensions become continuous, such that the theory appears to be seven-dimensional on the Higgs branch. There is however 
a peculiarity in this deconstruction which suggests that the strongly coupled Higgs branch theory is actually an eleven-dimensional gravitational theory: The deconstructed sevendimensional gauge theory has a UV completion in terms of M-theory on $A_{N-1}$. In the brane realization of the present deconstruction, M-theory on $A_{N-1}$ arises naturally in the continuum limit.

The Higgs branch of the quiver theory corresponds to the decoupling limit of D3-branes a finite distance away from the orbifold singularity. In the limit which we will consider the D3-branes probe an approximate $\mathbb{R}^{3} \times T^{3}$ geometry. The generation of three extra dimensions along the Higgs branch corresponds to T-dualizing along the three circular dimensions of the three-torus $T^{3}$, giving D6-branes wrapped on $T^{3}$. The seven-dimensional gauge theory living on the D6-branes does not decouple from the bulk degrees of freedom, such that the deconstructed theory is not just a seven-dimensional gauge theory. Due to a strong type IIA string coupling $g_{s}$ a better description is obtained by lifting to M-theory. The D6-branes in type IIA string theory lift to M-theory on an $A_{N-1}$ singularity. This suggests the equivalence of M-theory on $A_{N-1}$ with the continuum limit of the present quiver theory.

The equivalence is also supported by the following properties of the quiver theory on the Higgs branch. We find Kaluza-Klein states in the spectrum of massive gauge bosons which are responsible for the generation of three extra dimensions. Since in M-theory on $A_{N-1}$ the gauge theory is localized at the singularity which is a seven-dimensional submanifold, we can see three of the seven extra dimensions in the gauge boson spectrum. Moreover, we identify states in the spectrum of massive dyons which are identical to M2-branes wrapping two of the three compact extra dimensions.

The organization of this paper is as follows. In section 2 we will discuss the quiver theory and its Higgs branch. We will find a spectrum of massive gauge bosons and dyons indicating three extra dimensions and wrapped M2-branes. In section 3 we give stringtheoretical evidence for the equivalence of the continuum limit of the quiver theory with M-theory on $A_{N-1}$. In section 4 we conclude and discuss open problems such as the relation to matrix models.

\section{Deconstruction of M-theory on $T^{3} \times A_{N-1}$}

In this section we discuss the Higgs branch of the quiver theory, from which we deconstruct M-theory. The quiver theory is a four-dimensional non-supersymmetric field theory with gauge group $S U(N)^{N_{4} N_{6} N_{8}}$. This theory describes the decoupling limit of $N N_{4} N_{6} N_{8}$ D3-branes in type IIB string theory placed at a $\mathbb{C}^{3} / \Gamma$ orbifold singularity with $\Gamma \equiv \mathbb{Z}_{N_{4}} \times \mathbb{Z}_{N_{6}} \times \mathbb{Z}_{N_{8}}$. We argue that M-theory on an $A_{N-1}$ singularity is described by the orbifold model on the Higgs branch in a certain large $N_{4,6,8}$ and strong coupling limit.

\subsection{Non-supersymmetric orbifolds}

Before we discuss this particular product orbifold, let us briefly review non-supersymmetric orbifold models arising from $N$ D3-branes at $\mathbb{C}^{3} / \Gamma$. In order to break $\mathcal{N}=4$ supersymmetry of the parent theory down to $\mathcal{N}=0$, we choose $\Gamma$ to be a finite subgroup of the transverse 
$S U(4)$ isometry group. If $\Gamma \not \subset S U(3) \subset S U(4)$ then no supersymmetry is preserved. The orbifold action is embedded into the gauge group $U(N|\Gamma|)$.

The invariant components of the gauge field satisfy

$$
A_{\mu}=g(\xi) A_{\mu} g(\xi)^{-1}
$$

where $g(\xi)$ is the regular representation of the generator $\xi$ of $\Gamma$. The gauge field $A_{\mu}$ is a matrix in the adjoint representation of $U(N|\Gamma|)$. Since only block-diagonal matrices $A_{\mu}$ survive the projection, the gauge group is broken down to $U(N)^{|\Gamma|}$.

The four Weyl fermions of $\mathcal{N}=4$ super Yang-Mills theory transform in the 4 of $S U(4)$. Those fermions which are invariant under the orbifold must satisfy

$$
\psi^{i}=\xi^{a_{i}} g(\xi) \psi^{i} g(\xi)^{-1},
$$

where $i=1, \ldots, 4$ and

$$
a_{1}+a_{2}+a_{3}+a_{4} \equiv 0 \bmod |\Gamma|
$$

The complex scalars $\phi^{i}, i=1,2,3$, transform in the $\mathbf{6}$ of $S U(4)$, which can be obtained from the anti-symmetric tensor product of two 4's. Invariant scalars fulfill the conditions

$$
\phi^{i}=\xi^{b_{i}} g(\xi) \phi^{i} g(\xi)^{-1}
$$

where $b_{1}=a_{2}+a_{3}, b_{2}=a_{3}+a_{1}$, and $b_{3}=a_{1}+a_{2}$. A simple example of a nonsupersymmetric orbifold is $\mathbb{C} / \mathbb{Z}_{N^{\prime}}$ defined by the vectors

$$
a_{i}=(-1,1,1,-1), \quad b_{i}=(2,0,0) .
$$

Since $b_{2}=b_{3}=0$ the orbifold acts only in one of the three complex planes transverse to the D3-branes. The regular representation of the generator $\xi=e^{2 \pi i / N^{\prime}}$ of the group $\mathbb{Z}_{N^{\prime}}$ is given by $g(\xi)=\operatorname{diag}\left(I, \xi I, \xi^{2} I, \ldots, \xi^{N^{\prime}-1} I\right)$.

\subsection{D3-branes at $\mathbb{C}^{3} / \mathbb{Z}_{N_{4}} \times \mathbb{Z}_{N_{6}} \times \mathbb{Z}_{N_{8}}$ : orbifold realization of a non-supersym- metric quiver theory}

We now turn to the case where $N N_{4} N_{6} N_{8}$ D3-branes are placed at an orbifold $\mathbb{C}^{3} / \Gamma$ with $\Gamma \equiv \mathbb{Z}_{N_{4}} \times \mathbb{Z}_{N_{6}} \times \mathbb{Z}_{N_{8}}$. The orbifold action on the complex coordinates $z_{i}=(h, v, n)$ parametrizing $\mathbb{C}^{3}$ is given by

$$
h \rightarrow \xi_{4}^{2} h, \quad v \rightarrow \xi_{6}^{2} v, \quad n \rightarrow \xi_{8}^{2} n,
$$

where the generators of the groups $\mathbb{Z}_{N_{a}}$ are defined by $\xi_{a}=\exp \left(2 \pi i / N_{a}\right), a=4,6,8$. Each of the factors $\mathbb{Z}_{N_{a}}(a=4,6,8)$ acts on one of the three complex planes transverse to the stack of D3-branes. The orbifold action can be embedded into the subgroup $U(1)^{3}$ of the rotational group $S O(6)$.

The fields of the quiver theory descend from the $\mathcal{N}=4, d=4$ vector multiplet of the parent super Yang-Mills theory with gauge group $U\left(N N_{4} N_{6} N_{8}\right)$. We project out 
degrees of freedom which are not invariant under the orbifold group. The action of the product orbifold on the gauge field $A_{\mu}$, the three scalars $\phi^{i}=(h, v, n)$, and the four spinors $\psi^{i}=\left(\psi^{h}, \psi^{v}, \psi^{n}, \lambda\right)$ is given by

$$
\begin{aligned}
A_{\mu} & \rightarrow g(\xi) A_{\mu} g(\xi)^{-1}, \\
\phi^{i} & \rightarrow \xi_{4}^{b_{i}^{(4)}} \xi_{6}^{b_{i}^{(6)}} \xi_{8}^{b_{i}^{(8)}} g(\xi) \phi^{i} g(\xi)^{-1}, \\
\psi^{i} & \rightarrow \xi_{4}^{a_{i}^{(4)}} \xi_{6}^{a_{i}^{(6)}} \xi_{8}^{a_{i}^{(8)}} g(\xi) \psi^{i} g(\xi)^{-1},
\end{aligned}
$$

where $g(\xi)=g\left(\xi_{4}\right) \otimes g\left(\xi_{6}\right) \otimes g\left(\xi_{8}\right)$ is the regular representation of the generator $\xi=\xi_{4} \xi_{6} \xi_{8}$ of $\Gamma$. These transformation rules extend the invariance conditions given by Eqns. (2.1)-(2.4). For the vectors $a_{i}$ and $b_{i}$ we choose:

$$
\begin{array}{lll}
\mathbb{Z}_{N_{4}}: & a_{i}^{(4)}=(-1,1,1,-1), & b_{i}^{(4)}=(2,0,0), \\
\mathbb{Z}_{N_{6}}: & a_{i}^{(6)}=(1,-1,1,-1), & b_{i}^{(6)}=(0,2,0), \\
\mathbb{Z}_{N_{8}}: & a_{i}^{(8)}=(1,1,-1,-1), & b_{i}^{(8)}=(0,0,2) .
\end{array}
$$

Each of the three pairs of vectors $\left(a_{i}, b_{i}\right)$ gives rise to an orbifold $\mathbb{C} / \mathbb{Z}_{N}$ of the type given by Eq. (2.5). Together they define the non-supersymmetric orbifold $\mathbb{C}^{3} / \mathbb{Z}_{N_{4}} \times \mathbb{Z}_{N_{6}} \times \mathbb{Z}_{N_{8}}$. The vectors $b_{i}$ determine the action (2.6) on the coordinates $z_{i}=(h, v, n)$ via

$$
z_{i} \rightarrow \xi_{4}^{b_{i}^{(4)}} \xi_{6}^{b_{i}^{(6)}} \xi_{8}^{b_{i}^{(8)}} z_{i}
$$

The vectors $a_{i}$ give the corresponding action on the four fermions. The invariant fermions $\psi_{i, j, k}^{m}$ transform under the gauge group

$$
S U(N)^{N_{4} N_{6} N_{8}}
$$

as $\left(\mathrm{N}_{i, j, k}, \overline{\mathrm{N}}_{i \pm a_{m}^{(4)}, j \pm a_{m}^{(6)}, k \pm a_{m}^{(8)}}\right)$, where $\mathrm{N}_{i, j, k}\left(\overline{\mathrm{N}}_{i^{\prime}, j^{\prime}, k^{\prime}}\right)$ denotes the (anti-)fundamental representation of the gauge group $S U(N)_{i, j, k}\left(S U(N)_{i^{\prime}, j^{\prime}, k^{\prime}}\right)$. The invariant scalars $\phi_{i, j, k}^{m}$ transform as $\left(\mathrm{N}_{i, j, k}, \overline{\mathrm{N}}_{i \pm b_{m}^{(4)}, j \pm b_{m}^{(6)}, k \pm b_{m}^{(8)}}\right)$. We summarize the field content of our quiver theory in the following table:

\begin{tabular}{|l|l||l|l|}
\hline field & representation & field & representation \\
\hline$h_{i, j, k}$ & $\left(\mathrm{~N}_{i, j, k}, \overline{\mathrm{N}}_{i+2, j, k}\right)$ & $\psi_{i, j, k}^{h}$ & $\left(\mathrm{~N}_{i, j, k}, \overline{\mathrm{N}}_{i+1, j-1, k-1}\right)$ \\
$v_{i, j, k}$ & $\left(\mathrm{~N}_{i, j, k}, \overline{\mathrm{N}}_{i, j+2, k}\right)$ & $\psi_{i, j, k}^{v}$ & $\left(\mathrm{~N}_{i, j, k}, \overline{\mathrm{N}}_{i-1, j+1, k-1}\right)$ \\
$n_{i, j, k}$ & $\left(\mathrm{~N}_{i, j, k}, \overline{\mathrm{N}}_{i, j, k+2}\right)$ & $\psi_{i, j, k}^{n}$ & $\left(\mathrm{~N}_{i, j, k}, \overline{\mathrm{N}}_{i-1, j-1, k+1}\right)$ \\
$A_{i, j, k}^{\mu}$ & adjoint & $\lambda_{i, j, k}$ & $\left(\mathrm{~N}_{i, j, k}, \overline{\mathrm{N}}_{i+1, j+1, k+1}\right)$ \\
\hline
\end{tabular}

Table 1: Fields in the quiver theory and their transformation behaviour under the gauge group $S U(N)^{N_{4} N_{6} N_{8}}$.

The theory space is a three-dimensional lattice with $N_{4} N_{6} N_{8}$ sites which discretizes a three-dimensional torus $T^{3}$ as shown in Fig. 1. Each site represents one of the gauge groups 


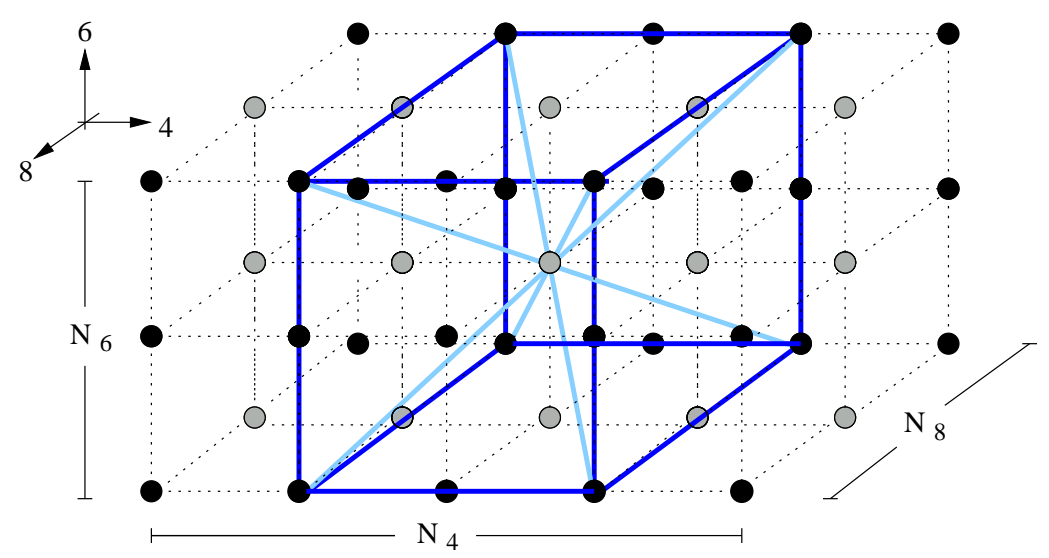

Figure 1: Theory space for the $\mathbb{Z}_{N_{4}} \times \mathbb{Z}_{N_{6}} \times \mathbb{Z}_{N_{8}}$ quiver theory. Dark and light (blue) lines in the basic cell represent bosonic and fermionic bifundamentals. Dotted lines are not physical and are just to guide the eye.

$S U(N)_{i, j, k}$ and its associated gauge boson $A_{i, j, k}^{\mu}$. Link fields start at a site $i, j, k$, where they transform in the fundamental representation $\mathrm{N}_{i, j, k}$, and end at a site $i^{\prime}, j^{\prime}, k^{\prime}$, where they transform in the antifundamental representation $\overline{\mathrm{N}}_{i^{\prime}, j^{\prime}, k^{\prime}}$. Fig. 11 shows the unit cell of the lattice spanned by the link fields. The bosonic bifundamentals $h_{i, j, k}, v_{i, j, k}, n_{i, j, k}$ (dark lines) form the edges of the unit cell, while the fermionic bifundamentals $\psi_{i, j, k}^{h}, \psi_{i, j, k}^{v}, \psi_{i, j, k}^{n}, \lambda_{i, j, k}$ (light lines) connect the corners with the centre of the cell. Translating the unit cell in the lattice we obtain a body-centred cubic lattice which is invariant under the 48 element octahedral symmetry group $O_{h}$. Such bcc lattices were also studied in the context of four-dimensional $\mathcal{N}=4$ super Yang-Mills theory on a three-dimensional lattice [24].

Let us now construct the Lagrangian for our orbifold model which consists of three parts,

$$
\mathcal{L}=\mathcal{L}_{\text {kin }}+\mathcal{L}_{\text {Yuk }}+\mathcal{L}_{\text {quartic }}
$$

It follows from the four-dimensional $\mathcal{N}=4$ super Yang-Mills theory with gauge group $U\left(N N_{4} N_{6} N_{8}\right)$ upon projecting out degrees of freedom which are not invariant under the orbifold group. The kinetic terms have the form

$$
\mathcal{L}_{\text {kin }} \supset \frac{1}{2} \operatorname{Tr}\left(D_{\mu} \varphi_{i^{\prime}, j^{\prime}, k^{\prime}}\right)^{\dagger} D^{\mu} \varphi_{i, j, k},
$$

where the field $\varphi_{i, j, k}$ is one of the seven bifundamentals transforming in the $\left(\mathrm{N}_{i, j, k}, \overline{\mathrm{N}}_{i^{\prime}, j^{\prime}, k^{\prime}}\right)$ as listed in Tab. 1. The covariant derivative of $\varphi_{i, j, k}$ is defined by

$$
D_{\mu} \varphi_{i, j, k}=\partial_{\mu} \varphi_{i, j, k}-i g A_{\mu}^{i, j, k} \varphi_{i, j, k}+i g \varphi_{i, j, k} A_{\mu}^{i^{\prime}, j^{\prime}, k^{\prime}} .
$$

We now consider the Yukawa and quartic scalar interactions $\mathcal{L}_{\text {Yuk }}$ and $\mathcal{L}_{\text {quartic }}$. Fig. 2 shows six of twelve possible triangles inside the basic cell. These triangles consist of two fermionic and one bosonic arrow and correspond to Yukawa couplings in the quiver theory. Each of the twelve triangles leads to a Yukawa term in the action. 


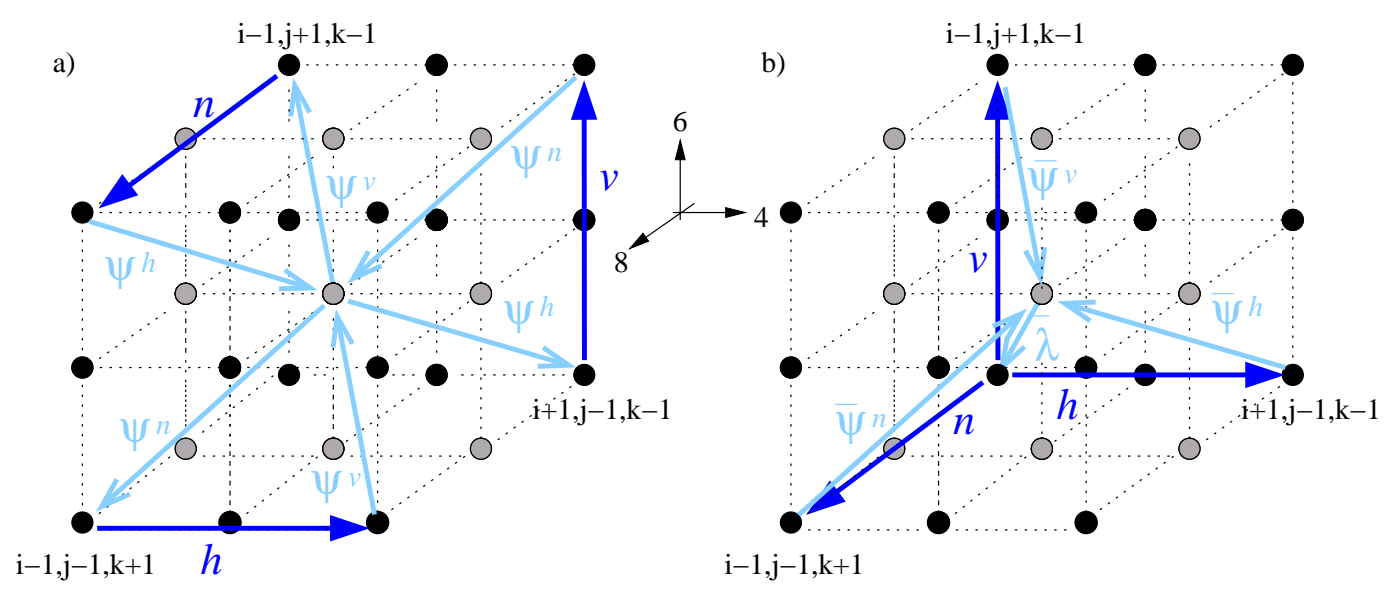

Figure 2: Oriented link fields in the basic cell. The site in the centre of the cell has labels $i, j, k$. The triangles represent possible Yukawa couplings in the quiver action.

For the Lagrangian $\mathcal{L}_{\text {Yuk }}$ we thus find

$$
\mathcal{L}_{\text {Yuk }}=\mathcal{L}_{\text {Yuk }}^{1}+\mathcal{L}_{\text {Yuk }}^{2}
$$

with

$$
\begin{aligned}
\mathcal{L}_{\text {Yuk }}^{1} & =i \sqrt{2} g \operatorname{Tr}\left(\psi_{i, j, k}^{v} n_{i-1, j+1, k-1} \psi_{i-1, j+1, k+1}^{h}-\psi_{i, j, k}^{n} v_{i-1, j-1, k+1} \psi_{i-1, j+1, k+1}^{h}\right. \\
& +\psi_{i, j, k}^{n} h_{i-1, j-1, k+1} \psi_{i+1, j-1, k+1}^{v}-\psi_{i, j, k}^{h} n_{i+1, j-1, k-1} \psi_{i+1, j-1, k+1}^{v} \\
& \left.+\psi_{i, j, k}^{h} v_{i+1, j-1, k-1} \psi_{i+1, j+1, k-1}^{v}-\psi_{i, j, k}^{v} h_{i-1, j+1, k-1} \psi_{i+1, j+1, k-1}^{n}+\text { c.c. }\right)
\end{aligned}
$$

and

$$
\begin{aligned}
\mathcal{L}_{\text {Yuk }}^{2} & =i \sqrt{2} g \operatorname{Tr}\left(\bar{\lambda}_{i, j, k} h_{i-1, j-1, k-1} \bar{\psi}_{i+1, j-1, k-1}^{h}-\bar{\psi}_{i, j, k}^{h} h_{i-1, j+1, k+1} \bar{\lambda}_{i+1, j+1, k+1}\right. \\
& +\bar{\lambda}_{i, j, k} v_{i-1, j-1, k-1} \bar{\psi}_{i-1, j+1, k-1}^{v}-\bar{\psi}_{i, j, k}^{v} v_{i+1, j-1, k+1} \bar{\lambda}_{i+1, j+1, k+1} \\
& \left.+\bar{\lambda}_{i, j, k} n_{i-1, j-1, k-1} \bar{\psi}_{i-1, j-1, k+1}^{n}-\bar{\psi}_{i, j, k}^{n} n_{i+1, j+1, k-1} \bar{\lambda}_{i+1, j+1, k+1}+\text { c.c. }\right)
\end{aligned}
$$

where summation over $i, j, k$ is understood. The terms with a positive sign in $\mathcal{L}_{\text {Yuk }}^{1}\left(\mathcal{L}_{\text {Yuk }}^{2}\right)$ correspond to the triangles in Fig. 2a(b) (those with negative sign are not shown). The Yukawa couplings in $\mathcal{L}_{\text {Yuk }}^{1}$ descend from the $\mathcal{N}=4$ superpotential $[H, V] N$, while those in $\mathcal{L}_{\text {Yuk }}^{2}$ come from the Kähler potential.

Quite analogously, squares in the quiver diagram represent quartic scalar terms which are given by ${ }^{1}$

$$
\begin{aligned}
\mathcal{L}_{\text {quartic }} & =g^{2} \operatorname{Tr}\left(n_{i, j, k} h_{i, j, k+2} \bar{n}_{i+2, j, k+2} \bar{h}_{i+2, j, k}-h_{i, j, k} n_{i+2, j, k} \bar{h}_{i+2, j, k+2} \bar{n}_{i, j, k+2}\right. \\
& +h_{i, j, k} v_{i+2, j, k} \bar{h}_{i+2, j+2, k} \bar{v}_{i, j+2, k}-v_{i, j, k} h_{i, j+2, k} \bar{v}_{i+2, j+2, k} \bar{h}_{i+2, j, k} \\
& \left.+v_{i, j, k} n_{i, j+2, k} \bar{v}_{i, j+2, k+2} \bar{n}_{i, j, k+2}-n_{i, j, k} v_{i, j, k+2} \bar{n}_{i, j+2, k+2} \bar{v}_{i, j+2, k}\right) .
\end{aligned}
$$

\footnotetext{
${ }^{1}$ There are some additional terms contributing to $\mathcal{L}_{\text {quartic }}$ : For brevity we did not list terms corresponding to degenerate squares coming from D-terms like $D \bar{h} h+D \bar{v} v+D \bar{n} n$.
} 
This model belongs to the class of conformal non-supersymmetric orbifold models studied in [25, 26]. In these models it is assumed that the orbifold group $\Gamma \subset S U(4)$ acts solely on the transverse space $\mathbb{C}^{3}$ of $M$ parallel D3-branes. Kachru and Silverstein [27] noticed that the orbifold group acts only on the $S^{5}$ factor of the near horizon geometry $A d S_{5} \times S^{5}$. In the AdS/CFT correspondence the isometry group of the $A d S_{5}$ space is identified with the conformal group of the field theory on its boundary. This implies classical conformal invariance of the worldvolume theory on the D3-branes. In [25] it was shown that if $M$ is finite and the regular representation of $\Gamma$ is chosen, the one-loop beta functions for the gauge couplings vanish in these theories. In the large $M$ limit one can even prove the vanishing of the beta functions to all orders in perturbation theory [26]. This holds for the present quiver theory which we consider in the limit $N_{4,6,8} \rightarrow \infty$ such that $M=N N_{4} N_{6} N_{8} \rightarrow \infty .^{2,3}$ Our non-supersymmetric quiver theory has therefore quantum conformal invariance. As discussed in detail in [6] conformal invariance guarantees that the quiver theory remains in the Higgs phase even at strong coupling.

A related question to that of conformal invariance is the stability of the moduli space. Since the theory is not supersymmetric the potential for the scalars is not necessarily protected against quantum corrections. This would change the moduli space of the classical theory. However, as shown in [26] all Feynman diagrams in the quiver theory are the same as in the $\mathcal{N}=4$ parent $S U(M)$ gauge theory up to possible $\frac{1}{M}$ corrections. In the large $M$ limit these corrections are suppressed and the potential remains unchanged. ${ }^{3}$ Although this points to a stable moduli space, we might still have troubles with divergencies coming from the twisted closed string sector.

The question of stability of our model is highly non-trivial. In contrast to supersymmetric orbifold models there are closed string tachyons in the twisted sector of nonsupersymmetric orbifolds of the type $\mathbb{C} / \mathbb{Z}_{N}$. The tachyon condensation leads to the decay of the orbifold as studied in 28]. The initial effect of the tachyons is to smooth out the orbifold singularity. An RG flow is initiated by the tachyon and the orbifold decays to flat space. If the initial state has been appropriately fine-tuned, the orbifold decay can take place in a series of transitions $\mathbb{C} / \mathbb{Z}_{N} \rightarrow \mathbb{C} / \mathbb{Z}_{N-2}$. For finite $N$ the orbifold becomes flat in a finite time. However, in the limit $N \rightarrow \infty$ the orbifold does not decay in a finite amount of time, since the orbifold goes through only finitely many transitions $\mathbb{C} / \mathbb{Z}_{N} \rightarrow \mathbb{C} / \mathbb{Z}_{N-2}$. The orbifold may however decay faster, e.g. via transitions $\mathbb{C} / \mathbb{Z}_{N} \rightarrow \mathbb{C} / \mathbb{Z}_{N-M}(M>2)$. If the quotient $M / N$ vanishes in the large $N$ limit the orbifold remains stable. In other words, the question is whether the flattening of spacetime induced by the tachyon condensation outweighs the extreme curvature at the singularity. We believe it does not and presume

\footnotetext{
${ }^{2}$ The basic requirement for vanishing beta function, Eq. (2.7) in [26], is satisfied: Let $\gamma^{a} \in \Gamma \equiv \mathbb{Z}_{N_{4}} \times$ $\mathbb{Z}_{N_{6}} \times \mathbb{Z}_{N_{8}}$ and $\gamma_{4,6,8}^{a} \in \mathbb{Z}_{N_{4,6,8}}$. Then

$$
\begin{aligned}
\operatorname{tr}\left(\gamma^{a}\right) & =\left(1+\gamma_{4}^{a}+\ldots+\left(\gamma_{4}^{a}\right)^{N_{4}-1}\right)\left(1+\ldots+\left(\gamma_{6}^{a}\right)^{N_{6}-1}\right)\left(1+\ldots+\left(\gamma_{8}^{a}\right)^{N_{8}-1}\right) N \\
& =\left\{\begin{array}{l}
N_{4} N_{6} N_{8} N \text { if } \gamma^{1}=1 \\
0 \forall \gamma^{a}, a \neq 1
\end{array}\right.
\end{aligned}
$$

${ }^{3}$ Note that it is not necessary to send $N \rightarrow \infty$ in order to take $M=N N_{4} N_{6} N_{8} \rightarrow \infty$.
} 
that our model is stable in the large $N_{4,6,8}$ limit. However, this issue deserves some further investigation.

Another essential feature in the deconstruction of M-theory is S-duality of the orbifold model. In 25] it was argued that in a conformal (non-supersymmetric) quiver theory the complex moduli $\tau_{i}$ are inherited from the coupling $\tau_{\text {par }}$ of the $\mathcal{N}=4$ parent theory (recall $\left.\tau_{i}=\tau_{\text {par }} /|\Gamma|\right)$. In the present quiver theory the $N_{4} N_{6} N_{8}$ gauge couplings $\tau_{i, j, k}$ associated with the gauge groups $S U(N)_{i, j, k}$ are all the same and related to $\tau_{\text {par }}$ by

$$
\tau \equiv \tau_{i, j, k}=\frac{\tau_{\mathrm{par}}}{N_{4} N_{6} N_{8}} .
$$

The strong-weak duality $g_{\text {par }} \rightarrow 1 / g_{\text {par }}$ thus amounts to an $S L(2, \mathbb{Z})$ S-duality $g \rightarrow N_{4} N_{6} N_{8} / g$ in the quiver theory.

\subsection{Generation of three compact extra dimensions in the low-energy effective field theory}

We now show by studying the mass spectrum of the gauge bosons that the quiver theory generates three circular extra dimensions at low energies. On the Higgs branch of the theory the scalar bifundamentals have diagonal expectation values,

$$
\left\langle h_{i, j, k}\right\rangle=v_{4}, \quad\left\langle v_{i, j, k}\right\rangle=v_{6}, \quad\left\langle n_{i, j, k}\right\rangle=v_{8},
$$

independent of $i, j, k$. These condensates break the gauge group $S U(N)^{N_{4} N_{6} N_{8}}$ down to the diagonal subgroup $S U(N)$. Upon substituting the vevs $v_{4}, v_{6}, v_{8}$, the scalar kinetic terms inside $\mathcal{L}_{\text {kin }}$ give rise to mass terms for the gauge bosons,

$$
\begin{aligned}
& \operatorname{Tr}\left|D^{\mu} h_{i, j, k}\right|^{2}=g^{2} v_{4}^{2}\left(A_{\mu}^{i, j, k}-A_{\mu}^{i+2, j, k}\right)^{2} \equiv A_{\mu}^{i, j, k}\left(\mathcal{M}_{4}\right)_{i i^{\prime}}^{2} \delta_{j j^{\prime}} \delta_{k k^{\prime}} A_{i^{\prime}, j^{\prime}, k^{\prime}}^{\mu} \\
& \operatorname{Tr}\left|D^{\mu} v_{i, j, k}\right|^{2}=g^{2} v_{6}^{2}\left(A_{\mu}^{i, j, k}-A_{\mu}^{i, j+2, k}\right)^{2} \equiv A_{\mu}^{i, j, k} \delta_{i i^{\prime}}\left(\mathcal{M}_{6}\right)_{j j^{\prime}}^{2} \delta_{k k^{\prime}} A_{i^{\prime}, j^{\prime}, k^{\prime}}^{\mu} \\
& \operatorname{Tr}\left|D^{\mu} n_{i, j, k}\right|^{2}=g^{2} v_{8}^{2}\left(A_{\mu}^{i, j, k}-A_{\mu}^{i, j, k+2}\right)^{2} \equiv A_{\mu}^{i, j, k} \delta_{i i^{\prime}} \delta_{j j^{\prime}}\left(\mathcal{M}_{8}\right)_{k k^{\prime}}^{2} A_{i^{\prime}, j^{\prime}, k^{\prime}}^{\mu}
\end{aligned}
$$

The matrices $\mathcal{M}_{4,6,8}$ have entries 2 on the diagonal and -1 on the second off-diagonal. As in [1] diagonalization of the mass matrices $\mathcal{M}_{4,6,8}$ yields the mass eigenvalues

$$
m_{4,6,8}^{k}=2 g v_{4,6,8} \sin \frac{2 \pi k}{N_{4,6,8}} \approx 2 g v_{4,6,8} \frac{2 \pi k}{N_{4,6,8}} \quad \text { for } k \ll N_{4,6,8} .
$$

For small enough $k$, this approximates the Kaluza-Klein spectrum of a seven-dimensional gauge boson compactified on a three-torus $T^{3}$ with radii $R_{4,6,8}$. The radii $R_{4,6,8}$ are fixed by the mass scales of the lightest KK modes $(k=1)$ which are given by

$$
m_{4,6,8}=\frac{1}{R_{4,6,8}}
$$

with

$$
2 \pi R_{4}=N_{4} a_{4}=\frac{N_{4}}{2 g v_{4}}, \quad 2 \pi R_{6}=N_{6} a_{6}=\frac{N_{6}}{2 g v_{6}}, \quad 2 \pi R_{8}=N_{8} a_{8}=\frac{N_{8}}{2 g v_{8}}
$$


and $a_{4,6,8}$ the lattice spacings. In principle, this KK spectrum is not protected and could receive quantum corrections at strong coupling. In a similar context |6| it was argued that such quantum corrections are proportional to $\frac{1}{N_{4,6,8}}$ and vanish in the large $N_{4,6,8}$ limit. Provided this is true, we explicitly deconstruct three compact extra dimensions with radii $R_{4,6,8}$.

The Higgs mechanism does induce masses both for the gauge bosons as well as for the bifundamental fermions and scalars. For instance, substituting vevs for the scalars inside the Lagrangian $\mathcal{L}_{\text {Yuk }}$ leads to fermionic mass terms. Such mass terms could in principle lead to a different mass spectrum due to the non-supersymmetric nature of our model. Following 29] one can however verify that the fermionic mass spectrum is identical to the gauge boson spectrum. Both the bosonic as well as the fermionic Kaluza-Klein spectra generate the same extra dimensions.

\subsection{M2-branes on the Higgs branch}

By studying the orbifold geometry we show in the next section that the Higgs branch theory is equivalent to M-theory on an $A_{N-1}$ singular geometry. We have seen that the Higgs branch theory contains seven-dimensional super Yang-Mills theory with gauge group $S U(N)$. In M-theory on $A_{N-1}$ the seven-dimensional $S U(N)$ gauge symmetry arises from M2-branes wrapping collapsed two-cycles at the singularity, see e.g. [30]. In other words, the deconstructed 7d super Yang-Mills theory is part of M-theory on $A_{N-1}$. However, M-theory contains more than just the $7 \mathrm{~d}$ gauge theory. We have to verify also the existence of M2- or M5-branes inside the quiver theory.

The states corresponding to M2-branes can be seen in the dyonic spectrum of the quiver gauge theory. The dyonic mass spectrum follows from that of the gauge bosons by S-duality. Substituting

$$
g \rightarrow \frac{N_{4} N_{6} N_{8}}{g}
$$

into Eq. (2.26), we find for the lowest dyonic states the masses

$$
M_{4}=8 \pi^{3} R_{6} R_{8} / g_{7}^{2}, \quad M_{6}=8 \pi^{3} R_{8} R_{4} / g_{7}^{2}, \quad M_{8}=8 \pi^{3} R_{4} R_{6} / g_{7}^{2},
$$

where the seven-dimensional coupling constant is $g_{7}^{2}=a_{4} a_{6} a_{8} g^{2}$. These masses are identical to those of two-branes wrapping around two-tori $T^{2}$ inside the $T^{3}$. We can read off the tension of the two-branes, $T_{2}=1 /(2 \pi)^{2} g_{7}^{2}$, which is identical to the tension of M2-branes, $T_{\mathrm{M} 2}=1 /(2 \pi)^{2} l_{p}^{3}$. This gives field-theoretical evidence that we really deconstruct M-theory. ${ }^{4}$

\subsection{Summary of the field theory results}

Let us summarize the properties of the $S U(N)^{N_{4} N_{6} N_{8}}$ quiver theory. We have seen that three extra dimensions with fixed radii $R_{4,6,8}$ are generated along its diagonal Higgs branch.

\footnotetext{
${ }^{4}$ We cannot see M5-branes in this way. The theory we expect to deconstruct is M-theory on the geometry $\mathbb{R}^{1,3} \times T^{3} \times A_{N-1}$. There are not enough compact dimensions inside this geometry which M5-branes could wrap around.
} 
For finite lattice spacings $a_{4,6,8}$ our four-dimensional quiver theory describes a sevendimensional theory with gauge coupling $g_{7}^{2}$ discretized on a three-dimensional toroidal lattice. Seven-dimensional super Yang-Mills theory breaks down at a certain cut-off $\Lambda_{7 \mathrm{~d}}$ above which it requires a UV completion. The cut-off of the deconstructed theory is given by the mass of the highest KK mode, $\Lambda=a^{-1}\left(a=\max \left[a_{4}, a_{6}, a_{8}\right]\right)$. In the continuum limit $a_{4,6,8} \rightarrow 0$, which requires $g \rightarrow \infty$ while keeping the radii $R_{4,6,8}$ and the seven-dimensional gauge coupling $g_{7}^{2}$ fixed, $\Lambda$ becomes very large, $\Lambda \gg \Lambda_{7 \mathrm{~d}}$. In the large $N_{4,6,8}$ limit we therefore expect to deconstruct not only $7 \mathrm{~d}$ super Yang-Mills theory but also its UV completion. This is shown schematically in Fig. 3. We show in the next section that the UV completion is M-theory on $A_{N-1}$ with Planck length $l_{p}^{3}=g_{7}^{2}$.

$$
\text { M-theory on } \mathrm{A}_{N-1}
$$

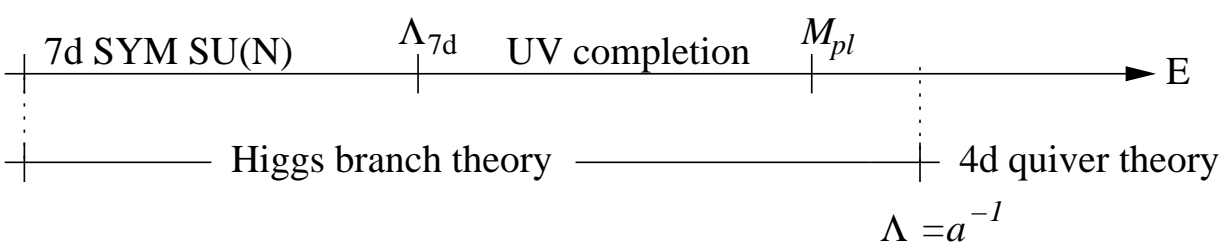

Figure 3: Various cut-offs in the deconstruction of M-theory.

M-theory on this geometry consists of two parts: The seven-dimensional gauge theory living on the singularity of the $A_{N-1}$ space couples to the eleven-dimensional bulk degrees of freedom of M-theory. In the gauge boson spectrum, we can therefore see only three of the seven string-theoretically predicted extra dimensions. It is not quite clear how the four dimensions of the $A_{N-1}$ space are generated. However, we have found a spectrum of massive dyons showing the presence of M2-branes in the deconstructed theory. This supports our conjecture that the deconstructed theory is M-theory on $A_{N-1}$.

\section{String-theoretical motivation for the equivalence}

We now motivate the conjecture of the last section by a string-theoretical analysis of the orbifold geometry. We show that supersymmetry with 16 supercharges is restored on the Higgs branch of our model, where the theory becomes M-theory on a $T^{3} \times A_{N-1}$ geometry. To this end, we consider the behaviour of the stack of D3-branes on the Higgs branch.

For this purpose, let us study the geometry of the orbifold in the vicinity of the D3branes which are located a distance $d$ away from the orbifold singularity. The product orbifold $\mathbb{C}^{3} / \mathbb{Z}_{N_{4}} \times \mathbb{Z}_{N_{6}} \times \mathbb{Z}_{N_{8}}$ can be parametrized by the complex coordinates $(i=1,2,3)$ 15

$$
z_{i}=r_{i} \exp \left(i \frac{b_{i}^{(4)}}{N_{4}} \theta_{1}+i \frac{b_{i}^{(6)}}{N_{6}} \theta_{2}+i \frac{b_{i}^{(8)}}{N_{8}} \theta_{3}\right) .
$$

For orthogonal vectors $b_{i}^{(4)}, b_{i}^{(6)}, b_{i}^{(8)}$, the orbifold metric $d s^{2}=d z_{i} d \bar{z}_{i}$ acquires the form

$$
d s^{2}=d \vec{r}^{2}+\frac{\left(\vec{r} \cdot \vec{b}^{(4)}\right)^{2}}{N_{4}^{2}} d \theta_{1}^{2}+\frac{\left(\vec{r} \cdot \vec{b}^{(6)}\right)^{2}}{N_{6}^{2}} d \theta_{2}^{2}+\frac{\left(\vec{r} \cdot \vec{b}^{(8)}\right)^{2}}{N_{8}^{2}} d \theta_{3}^{2},
$$


where $\vec{r}=\left(r_{1}, r_{2}, r_{3}\right), \vec{b}^{(4)}=\left(b_{1}^{(4)}, b_{2}^{(4)}, b_{3}^{(4)}\right)$, etc. The orbifold has the geometry of a threetorus fibration over $\mathbb{R}^{3}$ : We recover three circles $S^{1}$ parametrized by $\theta_{1,2,3} \in[0,2 \pi]$. For the particular choice of vectors $b_{i}$ as defined in Eqns. (2.10)-(2.12), the circles $S^{1}$ have radii $R_{S^{1}}=l_{s}^{2} / R_{4,6,8}$ with the radii $R_{4,6,8}$ given by

$$
R_{4}=\frac{N_{4} l_{s}^{2}}{2 d_{4}}, \quad R_{6}=\frac{N_{6} l_{s}^{2}}{2 d_{6}}, \quad R_{8}=\frac{N_{8} l_{s}^{2}}{2 d_{8}} .
$$

Here the D3-branes were assumed to be located at $\vec{r}=\left(d_{4}, d_{6}, d_{8}\right)$. Comparison with the radii defined in (2.27) yields relations between the parameters of the quiver theory $g, v_{4,6,8}$, $N_{4,6,8}$ and the string theory parameters $l_{s}, g_{s}, d_{4,6,8}=\left|z_{1,2,3}\right|, N_{4,6,8}$ :

$$
\frac{d_{4}}{N_{4} l_{s}^{2}}=\frac{2 \pi g v_{4}}{N_{4}}, \quad \frac{d_{6}}{N_{6} l_{s}^{2}}=\frac{2 \pi g v_{6}}{N_{6}}, \quad \frac{d_{8}}{N_{8} l_{s}^{2}}=\frac{2 \pi g v_{8}}{N_{8}} .
$$

These relations show that giving vevs $v_{4}, v_{6}, v_{8}$ to the scalar bifundamentals $h, v, n$ corresponds to moving the D3-branes a distance $d=\sqrt{d_{4}^{2}+d_{6}^{2}+d_{8}^{2}}$ away from the singularity. The continuum limit $a \rightarrow 0$ keeping $R_{4,6,8}$ fixed is obtained if we take $l_{s} \rightarrow 0, N_{4,6,8} \rightarrow \infty$ with $g_{s}=g^{2} / N_{4} N_{6} N_{8}$ and $d_{4,6,8} / N_{4,6,8} l_{s}^{2}$ fixed.

The orbifold may be visualized as a product of three cones. In the large $N_{4,6,8}$ limit each of the cones locally degenerates into a cylinder $\mathbb{R} \times S^{1}$ similar as in [6, 15]. The orbifold geometry in the vicinity of the D3-branes becomes approximately $\mathbb{R}^{3} \times T^{3}$ with $T^{3}$ a three-torus. Note that this induces a strong supersymmetry enhancement in the world volume theory. The $\mathcal{N}=4$ super Yang-Mills parent theory preserves 16 supercharges. The orbifold projection reduces supersymmetry to $\mathcal{N}=0$. Now in the large $N_{4,6,8}$ limit, the D3-branes probe the geometry $\mathbb{R}^{3} \times T^{3}$, which in contrast to the orbifold, does not break supersymmetry. On the Higgs branch the supersymmetry of the quiver theory is therefore enhanced again to 16 supercharges.

In the large $N_{4,6,8}$ limit the radii of the three circles $S^{1}$ are sub-stringy, i.e. $R_{S^{1}} \ll l_{s}$ if $R_{4,6,8} \gg l_{s}$, and the appropriate description is obtained by T-dualizing along the three directions of the torus 468. Details of the T-dualization are shown in the following table:

\begin{tabular}{|c|c|l|l|}
\hline duality & $\mathrm{D} 3$ & $l_{s} \rightarrow 0$ & $g_{s}$ fixed \\
\hline $\mathrm{T}$ in $x^{4}$ & $\mathrm{D} 4$ & $l_{s}^{\prime}=l_{s} \rightarrow 0$ & $g_{s}^{\prime}=g_{s} R_{4} / l_{s} \rightarrow \infty$ \\
$\mathrm{T}$ in $x^{6}$ & $\mathrm{D} 5$ & $l_{s}^{\prime \prime}=l_{s} \rightarrow 0$ & $g_{s}^{\prime \prime}=g_{s} R_{4} R_{6} / l_{s}^{2} \rightarrow \infty$ \\
$\mathrm{T}$ in $x^{8}$ & $\mathrm{D} 6$ & $l_{s}^{\prime \prime \prime}=l_{s} \rightarrow 0$ & $g_{s}^{\prime \prime \prime}=g_{s} R_{4} R_{6} R_{8} / l_{s}^{3} \rightarrow \infty$ \\
\hline M-theory & M-theory & $l_{p}^{3}=\left(l_{s}^{\prime \prime \prime}\right)^{3} g_{s}^{\prime \prime \prime}$ & $R_{11}=l_{s}^{\prime \prime \prime} g_{s}^{\prime \prime \prime}$ \\
lift & on $A_{N-1}$ & fixed & \\
\hline
\end{tabular}

Table 2: T-dualization in $x^{4,6,8}$ and lift to M-theory.

We started from D3-branes in the decoupling limit $l_{s} \rightarrow 0, g_{s}$ fixed. These D3-branes T-dualize to D6-branes which wrap a three-torus $T^{3}$ with large radii $R_{4,6,8}$. Due to a large string coupling $g_{s}^{\prime \prime \prime}$, a more appropriate description is obtained by lifting the stack of $N$ D6-branes to a singular $A_{N-1}$ geometry in M-theory. The seven-dimensional gauge theory located on the $A_{N-1}$ singularity has gauge coupling $g_{7}^{2}=l_{p}^{3}=\left(l_{s}^{\prime \prime \prime}\right)^{3} g_{s}^{\prime \prime \prime}=l_{s}^{2} R_{11}$. Since 
$R_{11}=g_{s} R_{4} R_{6} R_{8} / l_{s}^{2} \rightarrow \infty$ and $l_{s} \rightarrow 0$, we can hold both the gauge coupling $g_{7}$ and the eleven-dimensional Planck length $l_{p}$ fixed. The seven-dimensional gauge theory therefore does not decouple from the bulk gravity.

To conclude, string theory arguments suggest that our strongly coupled non-supersymmetric quiver theory on the Higgs branch describes M-theory on $T^{3} \times A_{N-1}$ in a large $N_{4,6,8}$ limit. Since M-theory reduces to eleven-dimensional supergravity at low-energies, we have deconstructed a gravitational theory!

\section{Conclusions}

We have deconstructed M-theory on a singular space of the type $T^{3} \times A_{N-1}$ from a fourdimensional non-supersymmetric quiver gauge theory with gauge group $S U(N)^{N_{4} N_{6} N_{8}}$. This theory is conformal in the large $N_{4,6,8}$ limit. We have given some evidence for the commutativity of the following diagram, which summarizes the deconstruction:

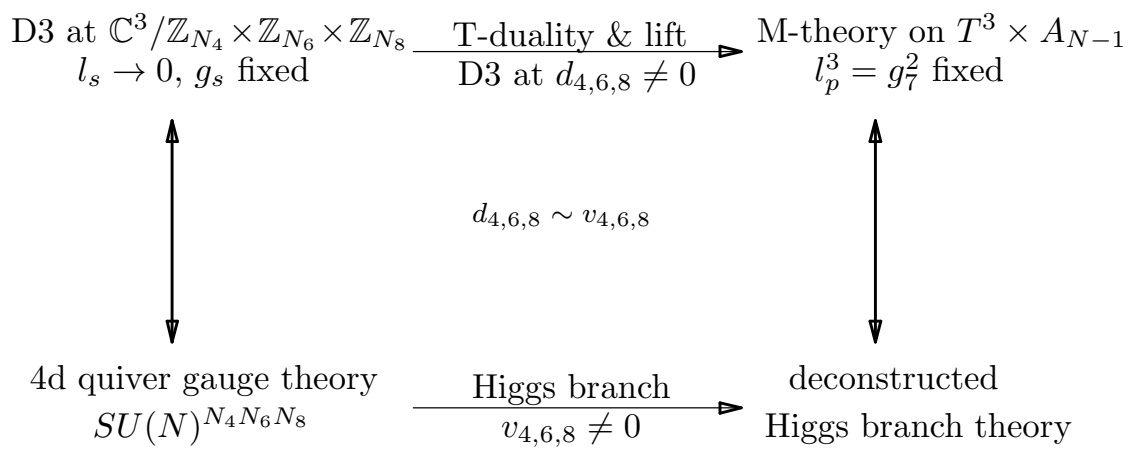

Figure 4: Orbifold realization of the quiver gauge theory and deconstruction of M-theory on $T^{3} \times A_{N-1}$.

The left hand side of the diagram shows the quiver gauge theory and its realization in type IIB string theory as a stack of D3-branes placed at the origin of an orbifold of the type $\mathbb{C}^{3} / \mathbb{Z}_{N_{4}} \times \mathbb{Z}_{N_{6}} \times \mathbb{Z}_{N_{8}}$. The right hand side represents the Higgs branch of the quiver theory and its corresponding realization in M-theory. Moving the D3-branes away from the orbifold singularity corresponds to the Higgs branch of the quiver theory. Exploiting string dualities, we map the geometry in the vicinity of the D3-branes in type IIB string theory to a $T^{3} \times A_{N-1}$ geometry in M-theory. We conclude that the deconstructed Higgs branch theory, in a particular strong coupling and large $N_{4,6,8}$ limit, is equivalent to M-theory on $T^{3} \times A_{N-1}$. This claim is further supported by a dyonic spectrum in the quiver theory which corrsponds to wrapped M2-branes. We thus provide a completely field-theoretical definition of M-theory on $T^{3} \times A_{N-1}$.

The deconstruction of M-theory does not suffer from the problems of the deconstruction of pure gravitational theories discussed in [19, 20, 21, 22, 23]. In quiver gauge theories the cut-off for the higher-dimensional behaviour of the theory can be taken to infinity.

However it would be of interest to find further field-theoretical evidence for the equivalence. For example, one would like to see how eleven-dimensional supergravity is realized 
in the model. The quiver model is formulated in terms of local fields of an ordinary fourdimensional Yang-Mills theory. An open question is the relation between these fields and the metric tensor or higher spin fields in M-theory. It would also be exciting to find a field-theoretical argument for the quiver theory to describe eleven dimensions besides the string-theoretical argument given in this paper.

The present $4 \mathrm{~d}$ quiver theory provides a non-perturbative definition of M-theory and might be an alternative to matrix models which describe the discrete light-cone quantization (DLCQ) of M-theory [31, 32]. It would be very interesting to find a relation between both approaches. The matrix model for M-theory on $T^{3} \times A_{N-1}$ is given by a $4 \mathrm{~d} \mathcal{N}=2$ super Yang-Mills quiver theory with gauge group $U(k)^{N}$ [33]. The parameter $k$ characterizes the discrete momentum $P_{-}=k / R$ of states in the lightlike direction. The Coulomb branch of this model describes the gauge theory located at the singularity of the geometry. The Higgs branch encaptures the physics of the spacetime away from the singularity. This matrix model has to be compared with the Higgs branch theory of our model, which has unbroken gauge group $S U(N)$ and preserves 16 supercharges in the continuum limit. The matrix model describes a sector of M-theory with fixed momentum $P_{-}$. In contrast, our model is not restricted on a particular sector of M-theory. Note however that the continuum limit $a_{4,6,8} \rightarrow 0$ requires one to consider the quiver theory at strong coupling, impeding perturbative access to M-theory.

\section{Acknowledgments}

We wish to thank James Babington, Ralph Blumenhagen, Karl Landsteiner, Esperanza Lopez and Dieter Lüst for useful discussions. The authors are particularly indebted to Johanna Erdmenger and Zachary Guralnik for helpful comments. The research of I.K. is funded by the DFG (Deutsche Forschungsgemeinschaft) within the Emmy Noether programme, grant ER301/1-2.

\section{References}

[1] N. Arkani-Hamed, A. G. Cohen and H. Georgi, Phys. Rev. Lett. 86 (2001) 4757 [arXiv:hep-th/0104005].

[2] C. T. Hill, S. Pokorski and J. Wang, Phys. Rev. D 64 (2001) 105005 [arXiv:hep-th/0104035].

[3] H. Georgi and A. Pais, Phys. Rev. D 10 (1974) 1246.

[4] M. B. Halpern and W. Siegel, Phys. Rev. D 11 (1975) 2967.

[5] M. R. Douglas and G. W. Moore, arXiv:hep-th/9603167.

[6] N. Arkani-Hamed, A. G. Cohen, D. B. Kaplan, A. Karch and L. Motl, JHEP 0301 (2003) 083 [arXiv:hep-th/0110146].

[7] C. Csaki, J. Erlich and J. Terning, Phys. Rev. D 67 (2003) 025019 [arXiv:hep-th/0208095].

[8] N. R. Constable, J. Erdmenger, Z. Guralnik and I. Kirsch, Phys. Rev. D 67 (2003) 106005 [arXiv:hep-th/0212136]. 
[9] N. R. Constable, J. Erdmenger, Z. Guralnik and I. Kirsch, Fortsch. Phys. 51 (2003) 732 [arXiv:hep-th/0212265].

[10] N. R. Constable, J. Erdmenger, Z. Guralnik and I. Kirsch, arXiv:hep-th/0211222.

[11] I. Rothstein and W. Skiba, Phys. Rev. D 65 (2002) 065002 [arXiv:hep-th/0109175].

[12] S. Mukhi, M. Rangamani and E. Verlinde, JHEP 0205 (2002) 023 [arXiv:hep-th/0204147].

[13] M. Alishahiha and M. M. Sheikh-Jabbari, Phys. Lett. B 538 (2002) 180 [arXiv:hep-th/0204174].

[14] J. Giedt, E. Poppitz and M. Rozali, JHEP 0303 (2003) 035 [arXiv:hep-th/0301048].

[15] P. Brax, R. A. Janik and R. Peschanski, Nucl. Phys. B 660 (2003) 194 [arXiv:hep-th/0303081].

[16] E. Poppitz, arXiv:hep-th/0306204.

[17] J. Dai and Y. S. Wu, arXiv:hep-th/0306216.

[18] A. Iqbal and V. S. Kaplunovsky, arXiv:hep-th/0212098.

[19] N. Arkani-Hamed and M. D. Schwartz, arXiv:hep-th/0302110.

[20] N. Arkani-Hamed, H. Georgi and M. D. Schwartz, arXiv:hep-th/ 0210184.

[21] M. D. Schwartz, arXiv:hep-th/0303114.

[22] V. Jejjala, R. G. Leigh and D. Minic, Phys. Lett. B 556 (2003) 71 [arXiv:hep-th/0212057].

[23] V. Jejjala, R. G. Leigh and D. Minic, arXiv:hep-th/0302230.

[24] D. B. Kaplan, E. Katz and M. Unsal, JHEP 0305 (2003) 037 [arXiv:hep-lat/0206019].

[25] A. E. Lawrence, N. Nekrasov and C. Vafa, Nucl. Phys. B 533 (1998) 199 [arXiv:hep-th/9803015].

[26] M. Bershadsky, Z. Kakushadze and C. Vafa, Nucl. Phys. B 523 (1998) 59 [arXiv:hep-th/9803076].

[27] S. Kachru and E. Silverstein, Phys. Rev. Lett. 80 (1998) 4855 [arXiv:hep-th/9802183].

[28] A. Adams, J. Polchinski and E. Silverstein, JHEP 0110 (2001) 029 [arXiv:hep-th/0108075].

[29] C. Csaki, J. Erlich, C. Grojean and G. D. Kribs, Phys. Rev. D 65 (2002) 015003 [arXiv:hep-ph/0106044].

[30] A. Sen, JHEP 9709 (1997) 001 [arXiv:hep-th/9707123].

[31] T. Banks, W. Fischler, S. H. Shenker and L. Susskind, Phys. Rev. D 55 (1997) 5112 [arXiv:hep-th/9610043].

[32] L. Susskind, arXiv:hep-th/9704080.

[33] O. J. Ganor and S. Sethi, JHEP 9801 (1998) 007 [arXiv:hep-th/9712071]. 